\title{
REPRESENTATION TYPE OF SCHUR SUPERALGEBRAS
}

\author{
DAVID J. HEMMER, JONATHAN KUJAWA, AND DANIEL K. NAKANO
}

\begin{abstract}
Let $S(m \mid n, d)$ be the Schur superalgebra whose supermodules correspond to the polynomial representations of the supergroup $G L(m \mid n)$ of degree $d$. In this paper we determine the representation type of these algebras (i.e. classify the ones which are semisimple, have finite, tame and wild representation type). Moreover, we prove that these algebras are in general not quasi-hereditary and have infinite global dimension.
\end{abstract}

\section{Introduction}

1.1 A central problem in the study of finite-dimensional algebras is to understand the structure of the indecomposable modules. As a first step one would like to know how many indecomposable modules a given algebra admits. Any finite-dimensional algebra can be classified into one of three categories: finite, tame, or wild representation type. A finitedimensional algebra $A$ has finite representation type if $A$ has finitely many indecomposable modules up to isomorphism. If $A$ is not of finite type then $A$ is of infinite representation type. Algebras of infinite representation type are either of tame representation type or of wild representation type. For algebras of tame representation type one has a chance of classifying all the indecomposable modules up to isomorphism.

There have been much progress in the past ten years in determining the representation type of important classes of finite-dimensional algebras. The Schur algebras and the $q$-Schur algebra are finite-dimensional algebras which arise in the representation theory of the general linear groups and symmetric groups. A complete classification of the representation type of these algebras was given by Doty, Erdmann, Martin and Nakano [E, DN, DEMN, EN1]. These algebras are fundamental examples of quasi-hereditary algebras (or equivalently highest weight categories) which were introduced by Cline, Parshall and Scott [CPS]. The blocks for the ordinary and parabolic BGG category $\mathcal{O}$ for finite-dimensional complex semisimple Lie algebras are other important examples of highest weight categories. Results pertaining to the representation type for these blocks were obtained independently by Futorny, Nakano and Pollack [FNP], and Brüstle, König and Mazorchuk [BKM] for the ordinary category

Date: October 2004.

1991 Mathematics Subject Classification. Primary 20C30.

Research of the first author was supported in part by NSF grant DMS-0102019.

Research of the second author was supported in part by NSF grant DMS-0402916.

Research of the third author was supported in part by NSF grant DMS-0400548. 
$\mathcal{O}$ and more recently for the parabolic category $\mathcal{O}$ by Boe and Nakano [BN]. For quasihereditary algebras the projective modules admit filtrations by certain standard modules. This information allows one to determine the structure of the projective modules which in turn can lead to an expression of the algebra via quiver and relations. In many cases this information can be used to determine the representation type of the algebra.

Other important classes of finite-dimensional algebras are quasi-Frobenius algebras where all projective modules are injective. These algebras all have infinite global dimension (except when the algebra is semisimple). For group algebras, restricted enveloping algebras, and Frobenius kernels homological information involving the theory of complexity and support varieties can be used to deduce information about the representation type of these algebras. For Hecke algebras of type $A$, the representation type of the blocks was given by Erdmann and Nakano in [EN2]. Ariki and Mathas [AM] classified the representation type for Hecke algebras of type $B$ using Fock space methods. Ariki $[A]$ recently has extended this classification to Hecke algebras of classical type.

The main result of this paper is a complete classification of the representation type for the Schur superalgebras $S(m \mid n, d)$. These algebras are of particular interest because the category of $S(m \mid n, d)$-supermodules is equivalent to the category of polynomial representations of degree $d$ for the supergroup $G L(m \mid n)$. Also, as with the classical Schur algebra, there is a Schur-Weyl duality between $S(m \mid n, d)$ and the symmetric group $\Sigma_{d}$ (see [BK]). We shall show that in most cases $S(m \mid n, d)$ is not quasi-hereditary, which is contrary to a recent conjecture given by Marko and Zubkov. Since the Schur superalgebras are not quasi-hereditary the aforementioned filtration techniques cannot be used. In order to obtain information about the basic algebra for certain Schur superalgebras we will compute the endomorphism algebras of direct sums of signed Young modules. This will entail knowing the structures of certain signed Young modules, which is of independent interest from the viewpoint of the representation theory of symmetric groups.

1.2 We review below the results for the ordinary Schur algebra $S(m, d)$, although the proof for $S(m \mid n, d)$ does not rely on these results. The representation type of $S(m, d)$ was determined in $[\mathrm{E}, \mathrm{DN}, \mathrm{DEMN}]^{1}$.

(1.2.1) Theorem . Let $S(m, d)$ be the Schur algebra over $k$ where char $k \geq 0$.

(a) $S(m, d)$ is semisimple if and only if one of the following holds

(i) $\operatorname{char} k=0$;

(ii) $d<p$;

(iii) $p=2, m=2, d=3$.

(b) $S(m, d)$ has finite representation type if and only if one of the following holds

(i) $p \geq 2, m \geq 3, d<2 p$;

(ii) $p \geq 2, m=2, d<p^{2}$;

(iii) $p=2, m=2, d=5,7$.

(c) $S(m, d)$ has tame representation type if and only if one of the following holds

(i) $p=3, m=3, d=7,8$;

\footnotetext{
${ }^{1}$ The statement that $S(2,11)$ for $p=2$ has tame representation type was inadvertently omitted from the original statement of the theorem [DEMN, Thm. 1.2(A)]. We thank Alison Parker for pointing this out to us.
} 
(ii) $p=3, m=2, d=9,10,11$;

(iii) $p=2, m=2, d=4,9,11$.

(d) In all other cases not listed above $S(m, d)$ has wild representation type.

The classification of the representation type of the $q$-Schur algebras $S_{q}(m, d)$ can be found in [EN1, Thm. 1.4(A)-(C)].

1.3 Throughout this paper we will assume that $k$ is an algebraically closed field of characteristic $p \geq 0$. The following theorem summarizes our results on the Schur superalgebras.

(1.3.1) Theorem . Let $S(m \mid n, d)$ be the Schur superalgebra over $k$ where char $k \neq 2$.

(a) $S(m \mid n, d)$ is semisimple if and only if one of the following holds

(i) $\operatorname{char} k=0$;

(ii) $d<p$;

(iii) $m=n=1$ and $p \nmid d$.

(b) $S(m \mid n, d)$ has finite representation type but is not semisimple if and only if one of the following holds

(i) $p \leq d<2 p$;

(ii) $m=n=1$ and $p \mid d$.

(c) In all other cases not listed above $S(m \mid n, d)$ has wild representation type.

We should remark that when in characteristic two $S(m \mid n, d)$ is equal to the ordinary Schur algebra $S(m+n, d)$, and its representation type is classified by Theorem 1.2.1.

\section{Comparing Module Categories for Superalgebras}

2.1 Recall that $k$ denotes our fixed ground field. All objects defined over $k$ (algebras, modules, superalgebras, supermodules, etc.) are assumed to be finite-dimensional as $k$ vector spaces.

A superspace is a $\mathbb{Z}_{2}$-graded $k$-vector space $V=V_{\overline{0}} \oplus V_{\overline{1}}$. Given a homogeneous element of a superspace, $v \in V$, we write $\bar{v} \in \mathbb{Z}_{2}$ for the degree of the element. If $V$ and $W$ are superspaces, then $\operatorname{Hom}_{k}(V, W)$ is naturally a superspace with $\operatorname{Hom}_{k}(V, W)_{\overline{0}}$ (resp. $\left.\operatorname{Hom}_{k}(V, W)_{\overline{1}}\right)$ consisting of all linear maps which preserve (resp. reverse) grading.

A superalgebra is a $\mathbb{Z}_{2}$-graded associative $k$-algebra $A=A_{\overline{0}} \oplus A_{\overline{1}}$ which satisfies $A_{r} A_{s} \subseteq$ $A_{r+s}$ for all $r, s \in \mathbb{Z}_{2}$. An $A$-supermodule is a $\mathbb{Z}_{2}$-graded $A$-module $M=M_{\overline{0}} \oplus M_{\overline{1}}$ which satisfies $A_{r} M_{s} \subseteq M_{r+s}$ for all $r, s \in \mathbb{Z}_{2}$. An $A$-supermodule homomorphism is a linear map $f: M \rightarrow N$ which satisfies $f(a m)=(-1)^{\bar{f}} \bar{a} a f(m)$ for all homogeneous $a \in A$ and all $m \in M$. As we will do later without comment, the given condition makes sense only for homogeneous elements. The general case is obtained by linearity. Note that if $M$ and $N$ are $A$-supermodules, then $\operatorname{Hom}_{A}(M, N)$ is naturally a superspace just as in the previous paragraph.

We can define the parity change functor $\Pi$ on the category of $A$-supermodules as follows. On an object $M$ we have that $\Pi M=M$ as a vector space, but we switch the $\mathbb{Z}_{2}$-grading by setting $(\Pi M)_{r}=M_{r+\overline{1}}$ for $r \in \mathbb{Z}_{2}$. The action of $A$ is defined via $a . m=(-1)^{\bar{a}} a m$ for homogeneous $a \in A$ and $m \in M$. On a morphism $f$, $\Pi f$ is the same linear map as $f$. We 
refer the reader to [BKI, Sect. 2] for further discussion of general results on superalgebras, supermodules, etc.

Throughout the remainder of this subsection we assume char $k \neq 2$. Given a superalgebra $A$ there are three module categories which are natural to consider:

(i) the category $A$-smod of all $A$-supermodules and all (not necessarily graded) $A$ supermodule homomorphisms;

(ii) the underlying even category of $A$-smod where we take the objects of $A$-smod but only the even (i.e. grading preserving) homomorphisms;

(iii) the category $A$-mod of all $A$-modules.

Observe that an $A$-supermodule is indecomposable or irreducible regardless of whether we view it as an object of the category $A$-smod or its underlying even category. If $M$ and $N$ are indecomposable $A$-supermodules and $f: M \rightarrow N$ is an $A$-supermodule isomorphism, write $f=f_{\overline{0}}+f_{\overline{1}}$ with $f_{r} \in \operatorname{Hom}_{A}(M, N)_{r}$ for $r \in \mathbb{Z}_{2}$. Note that by considering the $\mathbb{Z}_{2}$-grading it is straightforward to verify that $f_{\overline{0}}-f_{\overline{1}}: M \rightarrow N$ is also an isomorphism. Using this one can show that the map $M \oplus \Pi M \rightarrow N \oplus \Pi N$ given by

$$
(m, n) \mapsto\left(f_{\overline{0}}(m)+f_{\overline{1}}(n), f_{\overline{1}}(m)+f_{\overline{0}}(n)\right)
$$

is in fact an even isomorphism of $A$-supermodules. Consequently, by the graded version of the Krull-Schmidt theorem we see that $M$ is isomorphic to either $N$ or $\Pi N$ by an even isomorphism. Taken together the above discussion proves that the category $A$-smod is semisimple, finite, tame, or wild if and only if its underlying even category is semisimple, finite, tame, or wild, respectively. We use this observation without comment in what follows. The remainder of this section is devoted to studying the relationship between the representation type of these categories and the category $A$-mod.

Given a superspace $V$, let $\delta: V \rightarrow V$ be the linear involution defined by $\delta(v)=(-1)^{\bar{v}} v$, where $v \in V$ is homogeneous. In particular, the involution $\delta: A \rightarrow A$ is an algebra homomorphism and defines an action of $\mathbb{Z}_{2}$ on $A$. Consequently, we can define the smash product of $k \mathbb{Z}_{2}$ and $A$ as follows. Set

$$
B=k \mathbb{Z}_{2} \otimes_{k} A
$$

as a vector space. Identify $k \mathbb{Z}_{2}$ with $k[x] /\left(x^{2}-1\right)$. Then the product in $B$ is given by

$$
\left(x^{r} \otimes a\right)\left(x^{s} \otimes b\right)=x^{r+s} \otimes \delta^{s}(a) b,
$$

where $r, s \in\{0,1\}$ and $a, b \in A$. We identify $k \mathbb{Z}_{2}$ and $A$ as subalgebras via $k \mathbb{Z}_{2} \otimes 1$ and $1 \otimes A$, respectively. Note that since we assume char $k \neq 2, x$ acts semisimply on any $k \mathbb{Z}_{2}$-module with eigenvalues \pm 1 . The following result is proven in [Mui, Thm. 1.4b].

(2.1.2) Proposition. The category of B-modules is isomorphic to the underlying even category of A-supermodules.

Proof. Let $M$ be an $A$-supermodule. We define an action of $k \mathbb{Z}_{2}$ on $M$ via $x m=\delta(m)$. It is straightforward to verify that this makes $M$ into a $B$-module. Conversely, if $M$ is a $B$-module, then we can view it as an $A$-module by restriction. To obtain a $\mathbb{Z}_{2}$-grading we set $M_{\overline{0}}$ (resp. $M_{\overline{1}}$ ) to be the 1 (resp. -1) eigenspace of $x$ acting on $M$. One can verify that this $\mathbb{Z}_{2}$-grading makes $M$ into an $A$-supermodule. It is straightforward to verify that a morphism in one category restricts or extends to a morphism in the other category. 
2.2 Note that there is an involution $\zeta: B \rightarrow B$ given by $x^{r} \otimes a \mapsto(-1)^{r} x^{r} \otimes a$ for $r \in\{0,1\}$ and $a \in A$. Given a $B$-module $M$ we can twist it by $\zeta$, which we denote by $M^{\zeta}$. Namely, $M^{\zeta}=M$ as a vector space but with the action of $B$ given by $b . m=\zeta(b) m$ for all $b \in B$ and $m \in M$. Note that $M^{\zeta}$ is indecomposable if and only if $M$ is indecomposable.

Since $A$ is a subalgebra of $B$, there is a restriction functor $\operatorname{res}_{A}^{B}: B$-mod $\rightarrow A$-mod and an induction functor $\operatorname{ind}_{A}^{B}: A$-mod $\rightarrow B$-mod given by

$$
\operatorname{ind}_{A}^{B} N=B \otimes_{A} N
$$

for any $A$-module $N$. Note that

$$
\operatorname{ind}_{A}^{B} N \cong 1 \otimes N \oplus x \otimes N
$$

as $A$-modules. If $M$ is a $B$-module, then we have

$$
M \oplus M^{\zeta} \cong \operatorname{ind}_{A}^{B} \operatorname{res}_{A}^{B} M .
$$

Since $x$ acts semisimply on $M$ and $M^{\zeta}$, it suffices to define the isomorphism on eigenvectors $m \in M, m^{\prime} \in M^{\zeta}$ of eigenvalue $\varepsilon, \varepsilon^{\prime} \in\{ \pm 1\}$, respectively. Then the map $M \oplus M^{\zeta} \rightarrow$ $\operatorname{ind}_{A}^{B} \operatorname{res}_{A}^{B} M$ is given by

$$
\left(m, m^{\prime}\right) \mapsto(1+\varepsilon x) \otimes m+\left(1-\varepsilon^{\prime} x\right) \otimes m^{\prime} .
$$

To verify that the map is $B$-linear, one uses the observation that if $a \in A$ is an eigenvector for the action of $x$ with eigenvalue $\alpha$ and $m \in M$ is an eigenvector for the action of $x$ with eigenvalue $\beta$, then $a m$ is an eigenvector with eigenvalue $\alpha \beta$.

(2.2.3) Proposition . Let $M$ be an indecomposable $B$-module. Then $M$ is either indecomposable as an A-module or the direct sum of two indecomposable A-modules. Furthermore, every indecomposable A-module $N$ is a direct summand of $\operatorname{ind}_{A}^{B} N$ as $A$-modules.

On the other hand, if $M$ is an indecomposable $B$-module which contains $N$ as a direct summand, then $M$ is unique up to isomorphism and twisting by $\zeta$.

Proof. Let $M$ be an indecomposable $B$-module and let

$$
M=M_{1} \oplus \cdots \oplus M_{k}
$$

be a decomposition of $M$ into indecomposable $A$-modules. By using (2.2.2) and applying $\operatorname{ind}_{A}^{B}$ one has

$$
M \oplus M^{\zeta} \cong \operatorname{ind}_{A}^{B} M_{1} \oplus \cdots \oplus \operatorname{ind}_{A}^{B} M_{k}
$$

Since the left hand side is a decomposition as indecomposable $B$-modules, by the KrullSchmidt theorem it follows that (2.2.4) cannot have more than two direct summands.

Now say $N$ is an indecomposable $A$-module. By $(2.2 .1)$ we see that $N$ is a direct summand of $\operatorname{ind}_{A}^{B} N$. On the other hand, if $M$ is an indecomposable $B$-module which contains $N$ as a direct summand, then $\operatorname{ind}_{A}^{B} M \cong M \oplus M^{\zeta}$ contains $\operatorname{ind}_{A}^{B} N$. If $\operatorname{ind}_{A}^{B} N$ is indecomposable, then either $M \cong \operatorname{ind}_{A}^{B} N$ or $M \cong\left(\operatorname{ind}_{A}^{B} N\right)^{\zeta}$. If $\operatorname{ind}_{A}^{B} N$ is decomposable, then $\operatorname{ind}_{A}^{B} N \cong$ $M \oplus M^{\zeta}$. This proves the desired result.

As is well known, if $M$ is an irreducible $A$-supermodule then as an $A$-module $M$ is either irreducible or the direct sum of two irreducibles. Combining the previous result with Proposition 2.2.3 shows this behaviour extends to indecomposables. 
2.3 We can now prove the main result of this section: the representation type of $A$ as a superalgebra coincides with that of $A$ as an algebra.

(2.3.1) Theorem . The category A-smod is semisimple, finite, tame, or wild if and only if the same is true for A-mod.

Proof. By Proposition 2.1.2 it suffices to consider the category $B$-mod rather than $A$-smod.

Semisimple type: That $A$-smod is semisimple if and only if $B$-mod is semisimple is already known. See, for example, [BKI, Sect. 2] or [Mui, Thm. 1.4c].

Finite type: It is clear from Proposition 2.2.3 that $A$-mod has finitely many indecomposables if and only if $B$-mod does.

Tame type: Suppose $A$-mod has tame representation type. Let $t$ be an indeterminant and $d$ a positive integer. Then there are indecomposable $A$-modules $D_{d, 1}, \ldots, D_{d, r_{d}}$ and indecomposable $A-k[t]$-bimodules $E_{d, 1}, \ldots, E_{d, s_{d}}$ which are free as right $k[t]$-modules so that any indecomposable $A$-module of dimension $d$ is either isomorphic to $D_{d, i}$ for some $i$, or is isomorphic to $E_{d, i} \otimes_{k[t]} L$ for some $i$ and some irreducible $k[t]$-module $L$. Observe that the proof Proposition 2.2.3 applies verbatim to indecomposable $A-k[t]$-bimodules. Consequently, we can choose indecomposable $B$-modules $\widetilde{D}_{d, 1}, \ldots, \widetilde{D}_{d, r_{d}}$ and indecomposable $B-k[t]$-bimodules $\widetilde{E}_{d, 1}, \ldots, \widetilde{E}_{d, s_{d}}$ (which are free as $k[t]$-supermodules) where $\widetilde{D}_{d, i}$ contains $D_{d, i}$ as a direct summand as $A$-modules and $\widetilde{E}_{d, i}$ contains $E_{d, i}$ as a direct summand as $A-k[t]$-bimodules.

Let $M$ be an indecomposable $B$-module and $N$ an indecomposable direct summand of $M$ as an $A$-module. If $N \cong D_{d, i}$ for some $d$ and $i$, then by the uniqueness statement in Proposition 2.2.3 it follows that $M \cong \widetilde{D}_{d, i}$ or $M \cong\left(\widetilde{D}_{d, i}\right)^{\zeta}$. On the other hand, say $N \cong E_{d, i} \otimes_{k[t]} L$ for some $d, i$, and irreducible $k[t]$-module $L$. Since $E_{d, i}$ is a direct summand of $\widetilde{E}_{d, i}$, we have that $N \cong E_{d, i} \otimes_{k[t]} L$ is a direct summand of $\widetilde{E}_{d, i} \otimes_{k[t]} L$. However, by

[DEMN, Lemma 2.2] we know $\widetilde{E}_{d, i} \otimes_{k[t]} L$ is indecomposable. By uniqueness again we have $M \cong \widetilde{E}_{d, i} \otimes_{k[t]} L$ or $M \cong\left(\widetilde{E}_{d, i} \otimes_{k[t]} L\right)^{\zeta}=\left(\widetilde{E}_{d, i}\right)^{\zeta} \otimes_{k[t]} L$. Therefore, if $A$-mod is tame, then $B$-mod is tame.

Wild type: Suppose that $A$-mod has wild representation type. By [DEMN, Theorem 2.2] and Proposition 2.2.3 it follows that $B$-mod has wild representation type.

\section{Results on the Schur Superalgebra}

3.1 The Schur Superalgebra: Fix nonnegative integers $m, n$, and $d$, and fix a superspace $V$ with fixed basis $v_{1}, \ldots, v_{m+n}$ and $\mathbb{Z}_{2}$-grading given by setting $\bar{v}_{i}=\overline{0}$ for $i=1, \ldots, m$ and $\bar{v}_{i}=\overline{1}$ for $i=m+1, \ldots, m+n$. The tensor space $V^{\otimes d}$ is then naturally $\mathbb{Z}_{2}$-graded by setting the degree of $v_{i_{1}} \otimes \cdots \otimes v_{i_{d}}$ to be $\bar{v}_{i_{1}}+\cdots+\bar{v}_{i_{d}}$ for all $1 \leq i_{1}, \ldots, i_{d} \leq m+n$. The symmetric group $\Sigma_{d}$ acts on $V^{\otimes d}$ on the right via

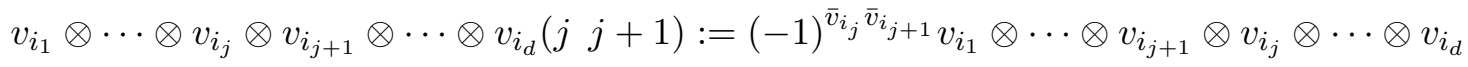


for $1 \leq i_{1}, \ldots, i_{d} \leq m+n$ and each $1 \leq j<d$.

The Schur superalgebra is defined to be

$$
S(m \mid n, d)=\operatorname{End}_{\Sigma_{d}}\left(V^{\otimes d}\right),
$$

with the $\mathbb{Z}_{2}$-grading given by setting $S(m \mid n, d)_{\overline{0}}$ (resp. $\left.S(m \mid n, d)_{\overline{1}}\right)$ to be the set of all degree preserving (resp. degree reversing) maps. Observe that when $p=2$ the above action coincides with the usual permutation action and so $S(m \mid n, d)=S(m+n, d)$.

We also note that $S(m \mid n, d) \cong S(n \mid m, d)$ as superalgebras. Namely, let $t:\{1, \ldots, m+$ $n\} \rightarrow\{1, \ldots, m+n\}$ be given by $t(i)=i+n$ if $i=1, \ldots, m$ and $t(i)=i-m$ if $i=$ $m+1, \ldots, m+n$. Fix a superspace $\widetilde{V}$ with fixed basis $\tilde{v}_{1}, \ldots, \tilde{v}_{m+n}$ and $\mathbb{Z}_{2}$-grading given by setting $\overline{\tilde{v}}_{i}=\overline{0}$ for $i=1, \ldots, n$ and $\overline{\tilde{v}}_{i}=\overline{1}$ for $i=n+1, \ldots, m+n$. Define a linear map $\tau: V^{\otimes d} \otimes \operatorname{sgn} \rightarrow \tilde{V}^{\otimes d}$ via

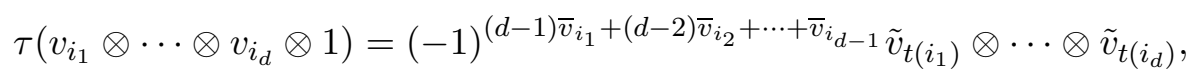

where $v_{i_{1}}, \ldots, v_{i_{d}}$ are elements of our fixed homogeneous basis for $V$. It is straightforward to verify on simple transpositions that in fact $\tau$ is a $k \Sigma_{d}$-module isomorphism (c.f. the proof of [BK, Theorem 6.4]). Consequently we have

$$
S(m \mid n, d)=\operatorname{End}_{\Sigma_{d}}\left(V^{\otimes d}\right) \cong \operatorname{End}_{\Sigma_{d}}\left(V^{\otimes d} \otimes \operatorname{sgn}\right) \cong \operatorname{End}_{\Sigma_{d}}\left(\widetilde{V}^{\otimes d}\right)=S(n \mid m, d),
$$

and the isomorphisms preserve the $\mathbb{Z}_{2}$-grading.

We now introduce certain modules which will be crucial in later calculations. Given a tuple of integers $\gamma=\left(\gamma_{1}, \ldots, \gamma_{t}\right)$ we denote the integer $\gamma_{1}+\cdots+\gamma_{t}$ by $|\gamma|$. Let

$$
\Lambda(m \mid n, d)=\left\{\left(\lambda_{1}, \ldots, \lambda_{m} \mid \mu_{1}, \ldots, \mu_{n}\right) \in \mathbb{Z}^{m+n} \mid \lambda_{i}, \mu_{i} \geq 0 \text { for all } i \text { and }|\lambda|+|\mu|=d\right\} .
$$

We write $(\lambda \mid \mu)$ for an element of $\Lambda(m \mid n, d)$.

In general, given $\gamma=\left(\gamma_{1}, \ldots, \gamma_{t}\right)$, a sequence of nonnegative integers summing to $r$, let

$$
\Sigma_{\gamma}=\Sigma_{\gamma_{1}} \times \Sigma_{\gamma_{2}} \times \cdots \times \Sigma_{\gamma_{t}}
$$

viewed as a subgroup of $\Sigma_{r}$ in the natural way by $\Sigma_{\gamma_{1}}$ acting as permutations of the first $\gamma_{1}$ letters, $\Sigma_{\gamma_{2}}$ acting as permutations of the next $\gamma_{2}$ letters, and so on.

Given $(\lambda \mid \mu) \in \Lambda(m \mid n, d)$ we define the signed permutation module $M^{(\lambda \mid \mu)}$ as

$$
M^{(\lambda \mid \mu)}=\operatorname{ind}_{\Sigma_{\lambda} \times \Sigma_{\mu}}^{\Sigma_{d}} k \otimes \operatorname{sgn},
$$

where $k$ denotes the trivial module for $\Sigma_{\lambda_{1}} \times \cdots \times \Sigma_{\lambda_{m}}$ and sgn denotes the one dimensional sign representation for $\Sigma_{\mu_{1}} \times \cdots \times \Sigma_{\mu_{n}}$.

By definition a $\Sigma_{d}$-module is a signed Young module if for some nonnegative integers $m$ and $n$ and $(\lambda \mid \mu) \in \Lambda(m \mid n, d)$ it is a direct summand of $M^{(\lambda \mid \mu)}$. Signed permutation modules and signed Young modules were first considered by Donkin [Do]. Finally we observe the well known fact that as $k \Sigma_{d}$ modules with the action from above we have:

$$
V^{\otimes d} \cong \bigoplus_{(\lambda \mid \mu) \in \Lambda(m \mid n, d)} M^{(\lambda \mid \mu)}
$$

3.2 We begin by collecting a few preliminary results. 
(3.2.1) Proposition . Let $S$ be a finite-dimensional algebra containing an idempotent e, so $e S e$ is a subalgebra of $S$.

(a) Every indecomposable eSe-module is a summand of the restriction to eSe of some indecomposable $S$-module.

(b) If eSe has infinite representation type then so does $S$.

(c) If eSe has wild representation type then so does $S$.

Proof. (a) Let $N$ be an indecomposable $e S e$-module. Let $U=S \otimes_{e S e} N$. Then $U$ is a nonzero $S$-module and:

$$
U \cong e U \oplus(1-e) U
$$

as $e S e$ modules. But $e U \cong N$. Now writing $U$ as a direct sum of indecomposable $S$ modules and an easy argument with the Krull-Schmidt theorem gives the result. (b) This is clear from part (a). If $S$ had only finitely many indecomposable modules then part (a) would imply $e S e$ does as well. (c) This statement follows from the argument of Theorem 2.2 in [DEMN]. If the statement of (a) holds for an arbitrary algebra $A$ and subalgebra $B$, the authors show that if $A$ is tame then so is $B$.

3.3 One can now apply the previous result to specific situation of $S(m \mid n, d)$ : This allows us to descend from the superalgebra $S\left(m^{\prime} \mid n^{\prime}, d\right)$ to $S(m \mid n, d)$ for $m \leq m^{\prime}, n \leq n^{\prime}$.

(3.3.1) Corollary . Let $m \leq m^{\prime}$ and $n \leq n^{\prime}$. Then:

(a) If $S(m \mid n, d)$ has infinite representation type then so does $S\left(m^{\prime} \mid n^{\prime}, d\right)$.

(b) If $S(m \mid n, d)$ has wild representation type then so does $S\left(m^{\prime} \mid n^{\prime}, d\right)$.

Proof. Recall that $\Lambda(m \mid n, d)$ is the set of pairs $(\lambda \mid \mu)$ where $\lambda$ and $\mu$ are compositions with $|\lambda|+|\mu|=d$ and $\lambda$ has at most $m$ parts, $\mu$ has at most $n$ parts. Thus $\Lambda(m \mid n, d)$ is naturally a subset of $\Lambda\left(m^{\prime} \mid n^{\prime}, d\right)$. Recall that:

$$
S\left(m^{\prime} \mid n^{\prime}, d\right) \cong \operatorname{End}_{k \Sigma_{d}}\left(\bigoplus_{(\lambda \mid \mu) \in \Lambda\left(m^{\prime} \mid n^{\prime}, d\right)} M^{(\lambda \mid \mu)}\right) .
$$

So if we let $e$ denote the projection onto the direct sum of all $M^{(\lambda \mid \mu)}$ with $(\lambda \mid \mu) \in$ $\Lambda(m \mid n, d)$ then it is clear that $e S\left(m^{\prime} \mid n^{\prime}, d\right) e \cong S(m \mid n, d)$, so we can apply Proposition 3.2.1 to prove the result.

3.4 The classical (and infinitesimal) Schur algebra $S(n, n)$ has a natural one-dimensional representation corresponding to the determinant for $G L(n)$. This allows one to embed the module category for $S(n, d)$ into that of $S(n, d+n)$, which can be used to study representation type (e.g. [DNP, Thm. 2.2]). Unfortunately the analogue of the determinant representation for the supergroup $G L(m \mid n)$, the one-dimensional Berezinian representation, is not polynomial and so it does not give a natural one-dimensional $S(m \mid n, m+n)$-module.

We instead use the existence of a one-dimensional representation for $S(2 \mid 1, p)$. Namely, let $L(0,0 \mid p)$ denote one-dimensional supermodule which is the Frobenius twist of the onedimensional $G L(2) \times G L(1)$-module $L(0,0) \otimes L(1)$ (see [BK, Remark 4.6]). Since the polynomial representations of $G L(m \mid n)$ correspond with the representations of the supermonoid 
$M(m \mid n)$ one can follow the arguments given in [DNP, Prop. 2.1, Prop. 2.2] by replacing the determinant representation with $L(0,0 \mid p)$ to deduce the following result.

(3.4.1) Proposition. If $S(2 \mid 1, d)$ has wild representation type then so does $S(2 \mid 1, d+c p)$ for any $c \in \mathbf{N}$.

3.5 We will assume familiarity with basic modular representation theory of the symmetric group. In the next section we will prove the main result of the paper. Surprisingly, we will use virtually no representation theory of the superalgebra. However, we will need to know that Young modules $Y^{\left(a, b, 1^{c}\right)}$ are actually signed Young modules when $c<p$ and $m=2, n=1$.

(3.5.1) Proposition . Let $1 \leq c<p$. Then the Young module $Y^{\left(a, b, 1^{c}\right)}$ is a summand of the signed permutation module $M^{(a, b \mid c)}$.

Proof. Since $c<p$ we have

$$
M^{\left(1^{c}\right)} \cong k \Sigma_{c} \cong \operatorname{ind}_{\Sigma_{1 c}}^{\Sigma_{c}} k \cong \bigoplus_{\lambda \vdash c}\left(S^{\lambda}\right)^{\operatorname{dim} S^{\lambda}} .
$$

But the Specht module $S^{\lambda}$ has dimension greater than one except when $\lambda=\left(1^{c}\right)$ or $\lambda=c$. Thus using (3.5.2) we obtain:

$$
M^{\left(a, b, 1^{c}\right)} \cong M^{(a, b, c)} \oplus M^{(a, b \mid c)} \bigoplus_{\lambda \vdash c, \lambda \neq\left(1^{c}\right),(c)} \operatorname{ind}_{\Sigma_{a} \times \Sigma_{b} \times \Sigma_{c}}^{\Sigma_{d}}\left(k \otimes k \otimes S^{\lambda}\right)^{d_{\lambda}}
$$

with all the $d_{\lambda}>1$. Since $Y^{\left(a, b, 1^{c}\right)}$ occurs exactly once as a direct summand of $M^{\left(a, b, 1^{c}\right)}$ and $\operatorname{Hom}_{\Sigma_{d}}\left(Y^{\left(a, b, 1^{c}\right)}, M^{(a, b, c)}\right)=0$, the result follows from (3.5.3).

3.6 The significance of Proposition 3.5.1 is that it gives us a Young module $Y^{\lambda}$ as a summand of the tensor space $V^{\otimes d}$, even though $\lambda$ has more than three parts and $m+n=3$. We need information about one other special signed Young module for the $p=3$ case.

(3.6.1) Proposition . Suppose $p$ does not divide a. Then:

$$
M^{(a, 0 \mid p)} \cong S^{\left(a+1,1^{p-1}\right)} \oplus S^{\left(a, 1^{p}\right)} .
$$

The two Specht modules in the decomposition are both irreducible and:

$$
S^{\left(a, 1^{p}\right)} \cong D^{\left(a, 2,1^{p-2}\right)} .
$$

Proof. The Specht module decomposition follows from Young's rule (see [J2, p.51]). The irreducibility of the "hook" Specht modules when $p \nmid d$ is well known work of Peel [P]. Finally knowing which irreducible $S^{\left(a, 1^{p}\right)}$ corresponds to can be calculated directly, or also follows as a very special case of a theorem of James [J1, Thm. A]. 


\section{Proof of the main result}

In this section we prove Theorem 1.3.1. One of the main ideas will be to identify a subalgebra $e S e$ with $k \mathcal{Q} / \mathcal{I}$ for some quiver $\mathcal{Q}$. Then we can use Gabriel's Theorem, since if the separated quiver is not a Dynkin or Euclidean diagram then the algebra is of wild type.

\section{1 $\mathrm{S}(\mathbf{1} \mid \mathbf{1}, \mathrm{d}):$}

If $n=0$ or $m=0$ the superalgebra $S(m \mid n, d)$ is isomorphic to the Schur algebra $S(m, d)$, so the smallest nontrivial case for us is the superalgebra $S(1 \mid 1, d)$. This has been studied by Marko and Zubkov:

(4.1.1) Proposition . [MZ, Prop. 2.1] Let $S=S(1 \mid 1, d)$. If $p$ does not divide d then $S$ is semisimple. If $p$ divides $d$ then $S$ has $d+1$ pairwise non-isomorphic one-dimensional modules, labelled by the weights $0,1, \ldots, d$. The algebra is basic, connected, and has left regular representation given by:

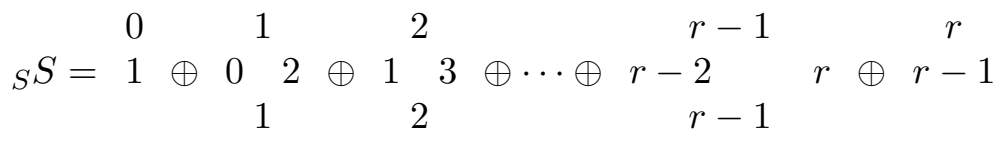

From here it is easy to obtain the quiver. In particular, for $m \geq 1$ we define an algebra $\tilde{\mathcal{A}}_{m}$ to be the algebra $k \mathcal{Q} / \mathcal{I}$ where $\mathcal{Q}$ is the quiver with $m+1$ vertices

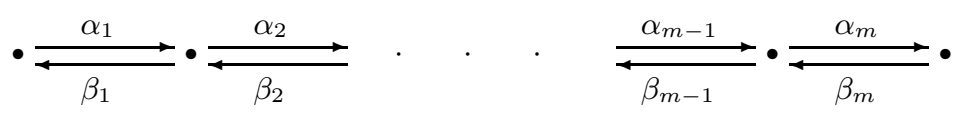

and $\mathcal{I}$ is the ideal generated by the relations:

$$
\alpha_{i} \alpha_{i+1}=0, \quad \beta_{i+1} \beta_{i}=0 \quad \alpha_{1} \beta_{1}=\beta_{m} \alpha_{m}=0 \text { and } \beta_{i} \alpha_{i}=\alpha_{i+1} \beta_{i+1} \text { for } 1 \leq i \leq m-1 .
$$

We remark that the quiver for $\tilde{\mathcal{A}}_{m}$ is the same as the quiver for Erdmann's algebra (defined in [E, 3.1]) $\mathcal{A}_{m+1}$ with one additional relation, namely that $\beta_{m} \alpha_{m}=0$. As we will see in Section 5, this extra relation is crucial in that it makes the algebra have infinite global dimension.

(4.1.3) Theorem . When $p$ divides $d$ the algebra $S(1 \mid 1, d)$ is isomorphic to the algebra $\tilde{\mathcal{A}}_{d}$ and has finite representation type.

Proof. The proof of the algebra isomorphism is essentially the same as Proposition 3.2 in [E], so we do not give it here. The algebra $\tilde{\mathcal{A}}_{m}$ is a three nilpotent algebra, but the only indecomposable modules of radical length three are projective. Hence, the representation type of $\tilde{\mathcal{A}}_{m}$ is the same as the representation type of $\tilde{\mathcal{A}}_{m}$ modulo its radical squared (which is two nilpotent). The separated quiver is a disjoint union of two copies of the Dynkin diagram $A_{m+1}$, and hence by Gabriel's Theorem the algebra is of finite type.

4.2 $\mathbf{S}(\mathbf{m} \mid \mathbf{n}, \mathbf{d}), \mathbf{d}<\mathbf{2} \mathbf{p}$ : We first consider the case where $p \leq d<2 p$. It turns out this case is essentially the same as for the Schur algebra $S(2, d)$. Recall that the summands of the signed permutation modules $M^{(\alpha \mid \beta)}$ are called signed Young modules. We recall the following theorem of Donkin. 
(4.2.1) Theorem . [Do, 2.3 (6)] Let $m, n \geq d$. The isomorphism classes of indecomposible signed Young modules are labelled by the set

$$
\Lambda^{++}(m \mid n, d):=\{(\lambda \mid p \mu)|| \lambda|+p| \mu \mid=d\}
$$

where $\lambda$ and $\mu$ are partitions.

Following [Do], we write $Y^{(\lambda \mid p \mu)}$ for the indecomposible signed Young module labelled by $(\lambda \mid p \mu) \in \Lambda^{++}(m \mid n, d)$.

We wish to show that for $d<2 p$ the signed Young modules are exactly the ordinary Young and twisted Young modules. To begin we will show that the nonprojective signed Young modules are all distinct.

Given a partition $\lambda \vdash d$, let $\lambda^{\prime}$ denote the transpose partition of $\lambda$. For $\lambda \vdash d p$-regular we let $m(\lambda)$ be the Mullineux conjugate of $\lambda$, so $D^{\lambda} \otimes \operatorname{sgn} \cong D^{m(\lambda)}$. It is well known that the Young module $Y^{m(\lambda)^{\prime}}$ is the projective cover of $D^{\lambda}$, a fact we will use several times below.

(4.2.2) Proposition. If the Young module $Y^{\lambda}$ is isomorphic to a twisted Young module $Y^{\mu} \otimes \operatorname{sgn}$, then $\lambda$ is p-restricted, in which case $\lambda=m\left(\mu^{\prime}\right)^{\prime}$.

Proof. Applying the adjoint Schur functor to $Y^{\lambda}$ and $Y^{\mu} \otimes \operatorname{sgn}($ see [HN, Thms 3.4.2, 3.8.2]) tells us that $P(\lambda) \cong T\left(\mu^{\prime}\right)$. But the tilting modules are self dual, so $P(\lambda)$ is self-dual and hence has simple socle $L(\lambda)$. But $V\left(\mu^{\prime}\right)$ is a submodule of $T\left(\mu^{\prime}\right)$ and the socle of $V\left(\mu^{\prime}\right)$ is $L\left(m\left(\mu^{\prime}\right)^{\prime}\right)$ so $\lambda=m\left(\mu^{\prime}\right)^{\prime}$ is $p$-restricted.

For $d<2 p$ it is clear that the $p$-singular partitions are exactly those of the form $\left(\mu_{1}+\right.$ $\left.p, \mu_{2}, \ldots\right)$ for $\mu$ a partition of $d-p$. Thus the number of signed Young modules for $d$ is the total number of partitions of $d$ plus the number of $p$-singular partitions, which by Prop. 4.2.2 is precisely the number of Young and twisted Young modules. We have shown the following result.

(4.2.3) Proposition. For $p \leq d<2 p$ the signed Young modules are exactly the set of Young modules $Y^{\lambda}$ together with the nonprojective twisted Young modules $Y^{\mu} \otimes$ sgn where $\mu$ is p-singular.

We also remark that the non-projective Young modules are actually irreducible and are in distinct blocks. Symmetric group blocks of defect one are well understood and we have the following. Let $\tau \vdash d-p$ and let $B$ be the block of $k \Sigma_{d}$ with $p$-core $\tau$. Let

$$
\lambda_{1}=\left(\tau_{1}+p, \tau_{2}, \ldots, \tau_{s}\right) \unrhd \lambda_{2} \unrhd \cdots \unrhd \lambda_{p}=\left(\tau, 1^{p}\right)
$$

be the $p$ partitions of $d$ with $p$-core $\tau$.

Let $\mu=\tau^{\prime}+(p)$. Then $\mu$ is $p$-singular and an easy calculation shows $Y^{\mu} \otimes \operatorname{sgn} \cong D^{\lambda_{p-1}}$, i.e. this is the nonprojective twisted Young module in the block. So the signed Young modules in the block are:

$$
Y^{\lambda_{1}} \cong D^{\lambda_{1}}, Y^{\lambda_{2}} \cong \begin{aligned}
& D^{\lambda_{1}} \\
& D^{\lambda_{2}} \\
& D^{\lambda_{1}}
\end{aligned}, Y^{\lambda_{i}} \cong \begin{gathered}
D^{\lambda_{i-1}} \\
D^{\lambda_{i}} D^{\lambda_{i-2}} \\
D^{\lambda_{i-1}}
\end{gathered}, Y^{\lambda_{p}} \cong \begin{aligned}
& D^{\lambda_{p-1}} \\
& D^{\lambda_{p-2}} \\
& D^{\lambda_{p-1}}
\end{aligned}, Y^{\mu} \otimes \operatorname{sgn} \cong D^{\lambda_{p-1}},
$$


where $3 \leq i<p$. Thus the basic algebra for $S(m \mid n, d)$ is a direct sum of two-sided ideals which are either semisimple (corresponding to blocks of $k \Sigma_{d}$ of defect 0 ), or which are isomorphic to

$$
\operatorname{End}_{k \Sigma_{d}}\left(\bigoplus_{i=1}^{p} Y^{\lambda_{i}} \oplus\left(Y^{\mu} \otimes \operatorname{sgn}\right)\right) .
$$

The quiver and relations for the algebra in (4.2.5) is just $\tilde{\mathcal{A}}_{p}$ which we saw earlier is of finite type. So we can now prove the first half of Theorem 1.3.1.

(4.2.6) Theorem . Let $S(m \mid n, d)$ be the Schur superalgebra where $0 \leq d<2 p$.

(a) $S(m \mid n, d)$ is semisimple if and only if one of the following holds

(i) $\operatorname{char} k=0$;

(ii) $d<p$;

(iii) $m=n=1$ and $p \nmid d$.

(b) $S(m \mid n, d)$ has finite representation type but is not semisimple if and only if one of the following holds

(i) $p \leq d<2 p$;

(ii) $m=n=1$ and $p \mid d$.

Proof. (a) If $d<p$ or char $k=0$ then $k \Sigma_{d}$ is semisimple, hence so is the algebra $S(m \mid n, d):=$ $\operatorname{End}_{k \Sigma_{d}}\left(V^{\otimes d}\right)$. Part (iii) follows by Proposition 4.1.1.

(b) The calculation above shows that $S(m \mid n, d)$ when $\max (m, n) \geq 2$ and $p \leq d<2 p$ has finite representation type (and is not semisimple). The same is true for $S(1 \mid 1, d)$ when $p \mid d$ by Proposition 4.1.1.

4.3 $\mathrm{S}(2 \mid 1, \mathrm{~d}), \mathrm{d} \geq 2 \mathrm{p}:$

In this section we begin to show that for $d \geq 2 p$ the algebra $S(2 \mid 1, d)$ has wild type. By Proposition 3.4.1 it will suffice to prove this for $2 p \leq d<3 p$. Recall that:

$$
S(2 \mid 1, d) \cong \operatorname{End}_{k \Sigma_{d}}\left(\bigoplus_{\left(\lambda_{1}, \lambda_{2} \mid \mu_{1}\right) \in \Lambda(2 \mid 1, d)} M^{\left(\lambda_{1}, \lambda_{2} \mid \mu_{1}\right)}\right) .
$$

In each case we will find some collection $\left\{Y_{i}\right\}$ of indecomposable signed Young modules such that the algebra $\operatorname{End}_{k \Sigma_{d}}\left(\oplus Y_{i}\right)$ is wild type. But this will imply $S(2 \mid 1, d)$ is wild type by Proposition 3.2.1.

To show that the algebra $\operatorname{End}_{k \Sigma_{d}}\left(\oplus Y_{i}\right)$ has wild type we will show, in each case, that it is isomorphic to an algebra $k \mathcal{Q} / \mathcal{I}$ where $\mathcal{Q}$ is a quiver and $\mathcal{I}$ is an ideal contained in the ideal generated by paths of length at least two. In each case the separated quiver of $\mathcal{Q}$ will not be a union of Dynkin or extended Dynkin diagrams, which by Gabriel's theorem means the algebra is of wild type. The main difficulty will be to make an appropriate choice of the $\left\{Y_{i}\right\}$.

Our proof will split into three cases; each following the same logic. First we handle the case $2 p \leq d<3 p-2$. Then we handle $d=3 p-2$ and $d=3 p-1$ separately. That this happens is not surprising, as the blocks of defect two are slightly different in the three cases, see for instance Table 1 in $[S]$. Essentially everything is understood for symmetric group 
blocks of defect two. The decomposition numbers are all known by [R]. The structure of the projective indecomposable modules can be determined from [S]. Finally Chuang and Tan $[\mathrm{CT}]$ have determined the structure of the nonprojective Young modules. Thus we just need to judiciously select signed Young modules to get a quiver of wild type. We will not give the details of determining the module structures we need, they can be found in the works cited above.

Recall that if $\lambda$ is a $p$-regular partition of $d$, we write $m(\lambda)$ for the Mullineux conjugate of $\lambda$, so $D^{\lambda} \otimes \operatorname{sgn} \cong D^{m(\lambda)}$ and the Young module $Y^{m(\lambda)^{\prime}}$ is the projective cover of $D^{\lambda}$.

$4.4 \mathbf{S}(\mathbf{2} \mid \mathbf{1}, \mathbf{d}), \mathbf{2} \mathbf{p} \leq \mathbf{d}<\mathbf{3 p}-\mathbf{2}$ : Let $d=2 p+t$ for $0 \leq t<p-2$. Using the Chuang-Tan notation we have the following partitions in the principal block of $\Sigma_{d}$ :

$$
\lambda^{(0)}=(2 p+t), \quad \lambda^{(1)}=(2 p-1, t+1), \quad \lambda^{(2)}=(2 p-2, t+1,1), \quad \lambda^{(*)}=(p+t, p) .
$$

If we let $\sigma=(p+t, p-1,1)$ then $\sigma$ is $p$-restricted and $Y^{\sigma}$ is the projective cover of $D^{\lambda^{(1)}}$. Using $i$ to denote $D^{\lambda^{(i)}}$ we have the following Loewy structures [CT, Thm. 2.4]:

$$
\begin{aligned}
& Y^{\lambda^{(0)}} \cong 0, \quad Y^{\lambda^{(1)}} \cong \begin{array}{l}
0 \\
1 \\
0
\end{array}, \quad Y^{\lambda^{(2)}} \cong \begin{array}{c}
1 \\
0 \\
1
\end{array} 2, \quad Y^{\lambda^{(*)}} \cong 0_{1}^{1} * \\
& Y^{\sigma} \cong \begin{array}{ccc} 
& 1 & \\
* & 0 & 2 \\
1 & \mu & 1 \\
* & 0 & 2 \\
& 1
\end{array} .
\end{aligned}
$$

Since all the partitions above have third part zero or one and since by Proposition 3.5.1 $Y^{(a, b, 1)}$ is a summand of $M^{(a, b \mid 1)}$, we know all the modules in (4.4.1) are signed Young modules for $m=2, n=1$. Consider the following quiver $\mathcal{L}$ given below:

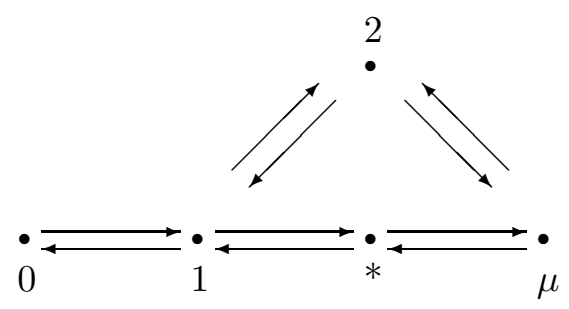

Let $U$ be the direct sum of the five modules in (4.4.1). We have:

\section{(4.4.2) Theorem .}

(a) The algebra $\operatorname{End}_{k \Sigma_{2 p+t}}(U)$ is isomorphic to the algebra $k \mathcal{L} / \mathcal{I}$ where $I$ is in the ideal generated by paths of length two. In particular it is of wild representation type.

(b) The superalgebra $S(2 \mid 1, d)$ has wild representation type for $2 p \leq d<3 p-2$. 
Proof. (a) The signed Young modules making up U have the same structure as the modules $E_{i}$ for $1 \leq i \leq 5$ in Prop. 3.10 of [E] and the determination of the quiver proceeds in exactly the same way.

(b) This follows immediately from part (a) by calculating the separated quiver of $\mathcal{L}$. The separated quiver is two copies of:

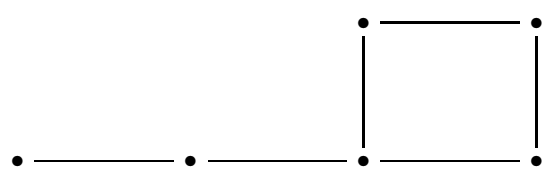

which is not a Dynkin or Euclidean diagram.

4.5 $\mathbf{S}(\mathbf{2} \mid \mathbf{1}, \mathbf{3} \mathbf{p}-\mathbf{2})$ : Let $d=3 p-2$. Using the Chuang-Tan notation we have the following partitions in the principal block of $\Sigma_{d}$ :

$$
\begin{gathered}
\lambda^{(0)}=(3 p-2), \quad \lambda^{(1)}=(2 p-1, p-1), \quad \lambda^{(2)}=\left(2 p-2, p-2,1^{2}\right), \\
\lambda^{(3)}=\left(2 p-2, p-3,1^{3}\right), \quad \lambda^{(*)}=(2 p-2, p), \quad \delta=(2 p-2, p-1,1) .
\end{gathered}
$$

It is also an easy calculation that:

$$
m(\delta)^{\prime}=\left(2 p-3, p-1,1^{2}\right)
$$

so $Y^{\left(2 p-3, p-1,1^{2}\right)}$ is the projective cover of $D^{\delta}$. Let $\tau=\left(2 p-3, p-1,1^{2}\right)$. Using $i$ to denote $D^{\lambda^{(i)}}$ we have the following structures for Young modules [CT]:
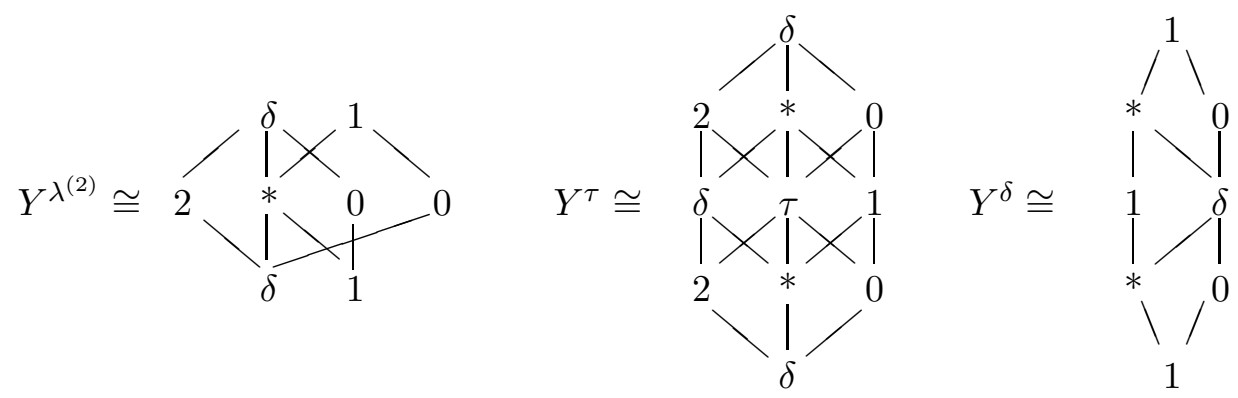

$$
Y^{\lambda^{(0)}} \cong 0, \quad Y^{\lambda^{(1)}} \cong \begin{aligned}
& 0 \\
& 1 \\
& 0
\end{aligned}, \quad Y^{\lambda^{(3)}} \cong \begin{gathered}
2 \\
2
\end{gathered} 3 .
$$

All the Young modules in (4.5.1) are either for two-part partitions or partitions of the form $\left(a, b, 1^{c}\right)$ for $c<p$, so by Proposition 3.5.1 they are all signed Young modules. Now consider the following quiver which we denote by $\mathcal{Q}$. 


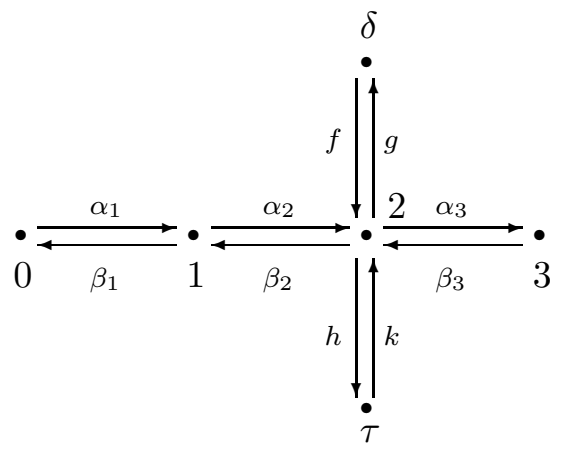

Let $T$ be the direct sum of the six signed Young modules in (4.5.1). Then we have the following result.

\section{(4.5.2) Theorem .}

(a) The algebra $\Lambda=\operatorname{End}_{k \Sigma_{3 p-2}}(T)$ is isomorphic to the algebra $k \mathcal{Q} / \mathcal{I}$ where $\mathcal{I}$ is the ideal generated the relations:

$$
\begin{gathered}
\alpha_{1} \alpha_{2}=\alpha_{2} \alpha_{3}=\alpha_{2} h=\beta_{3} \beta_{2}=\beta_{2} \beta_{1}=\beta_{3} g=f \alpha_{3}=\alpha_{2} h=0 \\
h k=g f=\beta_{1} \alpha_{2}+\alpha_{3} \beta_{3}
\end{gathered}
$$

(b) The superalgebra $S(2 \mid 1, d)$ has wild representation type for $d=3 p-2$.

Proof. (a) Let $\left\{e_{0}, e_{1}, e_{2}, e_{3}, e_{\delta}, e_{\tau}\right\} \in \Lambda$ be the canonical projections onto the Young module summands of $T$. Choose nonzero maps $\alpha_{i}$ and $\beta_{i}$. (The module structures leave no choice for the kernels and images of these maps). The image and kernels of the maps $f, g, h, k$ can also be determined by the structure of the modules given in (4.5.1). In particular we have the following Loewy structures for the kernels (from which the images are easily determined):

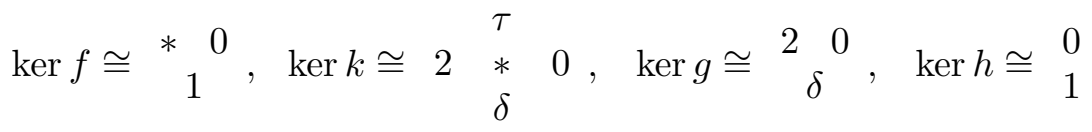

Knowing the kernels and images of the maps is sufficient to check that the zero relations in (4.5.3) are satisfied. We need only show we can choose the maps so that $h k=g f=$ $\beta_{1} \alpha_{2}+\alpha_{3} \beta_{3}$.

Notice that the image of $\beta_{2} \alpha_{2}$ is the simple module 1 and the image of $\alpha_{3} \beta_{3}$ is the simple module $\delta$ while both $h k$ and $g h$ have image equal to the socle of $Y^{\lambda^{(2)}}$. Since $\operatorname{End}\left(Y^{\lambda^{(2)}}\right)$ is three-dimensional, we can take as a basis $\left\{e_{2}, \alpha_{3} \beta_{3}, \beta_{2} \alpha_{2}\right\}$. Write:

$$
\begin{aligned}
h k & =a \beta_{2} \alpha_{2}+b \alpha_{3} \beta_{3} \\
g f & =c \beta_{2} \alpha_{2}+c \alpha_{3} \beta_{3}
\end{aligned}
$$

where the images ensure $a, b, c, d$ are all nonzero.

Now just do the substitutions:

$$
\beta_{2}^{\prime}=a \beta_{2}, \alpha_{2}^{\prime}=c \alpha_{2}, \beta_{3}^{\prime}=b \beta_{3}, \alpha_{3}^{\prime}=d \alpha_{4} .
$$


This will give the desired relation without effecting the earlier zero relations. The maps $\left\{\alpha_{i}, \beta_{i}, h, k, f, g\right\}$ generate $\operatorname{rad} \Lambda$ and are independent modulo $I^{2}$, so there is an epimorphism $k \mathcal{Q} / \mathcal{I}$ onto $\Lambda$. Now one can simply check the dimensions agree to obtain the isomorphism.

(b) This follows immediately from (a) by calculating the separated quiver of $\mathcal{Q}$ and verifying that it is not a Dynkin or Euclidean diagram.

In the special case where $p=3$ (i.e. $S(2 \mid 1,7)$ ) the partition $\lambda^{(3)}$ above does not exist. In this case to obtain the same quiver just replace $Y^{\lambda^{(3)}}$ by the irreducible module $D^{\delta}$, which is a signed Young module by Proposition 3.6.1. The quiver and relations are easily seen to be identical.

\section{6 $\mathrm{S}(2 \mid \mathbf{1}, 3 \mathrm{p}-1)$ :}

Let $d=3 p-1$. Using the Chuang-Tan notation we have the following partitions in the principal block of $\Sigma_{d}$ :

$$
\begin{gathered}
\lambda^{(0)}=(3 p-1), \quad \lambda^{(1)}=(2 p-1, p-1,1), \quad \lambda^{(2)}=\left(2 p-1, p-2,1^{2}\right), \\
\lambda^{(3)}=\left(2 p-1, p-3,1^{3}\right), \quad \lambda^{(*)}=(2 p-1, p), \quad \rho=(2 p-2, p, 1),
\end{gathered}
$$

where $\rho=m\left(\lambda^{(*)}\right)^{\prime}$, and hence $Y^{\rho}$ is the projective cover of $D^{\lambda^{(*)}}$.

Using $i$ to denote $D^{\lambda^{(i)}}$ we have the following structures for Young modules [CT]:

$$
\begin{aligned}
& Y^{\lambda^{(0)}} \cong 0, \quad Y^{\lambda^{(*)}} \cong *, \quad Y^{\lambda^{(1)}} \cong \begin{array}{c}
0 \\
0 \\
0 *
\end{array} \quad, \quad Y^{\lambda^{(2)}} \cong \begin{array}{c}
1 \\
0
\end{array} \\
& Y^{\lambda^{(3)}} \cong{ }_{1}^{2} 3, \quad Y^{\rho} \cong * \begin{array}{l}
* \\
1 \\
0 \\
1 \\
*
\end{array}
\end{aligned}
$$

Now consider the following quiver called $\mathcal{H}$ :

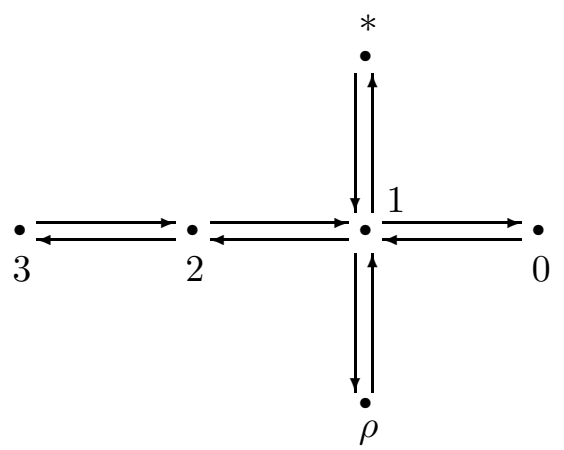

Let $W$ be the direct sum of the six signed Young modules in (4.6.1). Then we have the following result. 


\section{(4.6.2) Theorem .}

(a) The algebra $\operatorname{End}_{k \Sigma_{3 p-1}}(W)$ is isomorphic to the algebra $k \mathcal{H} / \mathcal{I}$ where $\mathcal{I}$ is in the ideal generated by paths of length two. In particular it is of wild representation type.

(b) The superalgebra $S(2 \mid 1, d)$ has wild representation type for $d=3 p-1$.

Proof. (a) We leave the calculations here for the reader. They are similar to the proof of Theorem 4.5.2. (b) Once again the separated quiver for $\mathcal{H}$ is not Dynkin or Euclidean so $S(2 \mid 1,3 p-1)$ is of wild representation type.

Just as in the $3 p-2$ case, we remark that the partition $\lambda^{(3)}$ does not exist when $p=3$ (i.e. $S(2 \mid 1,8))$. Again, replacing $Y^{\lambda^{(3)}}$ with the simple module $D^{(5,2,1)} \cong D^{\lambda^{(1)}}$ gives the same quiver. $D^{(5,2,1)}$ is a signed Young module by Proposition 3.6.1.

4.7 We can now complete the proof of Theorem 1.3.1 by proving the following result.

(4.7.1) Theorem . The algebra $S(m \mid n, d)$ has wild representation type when $\max (m, n) \geq 2$ and $d \geq 2 p$.

Proof. Since $S(m \mid n, d) \cong S(n \mid m, d)$ we may assume that $m \geq n$. By assumption $m \geq$ 2 and $n \geq 1$. The algebra $S(2 \mid 1, d), 2 p \leq d<3 p$ has wild representation type from Theorems 4.4.2(b), 4.5.2(b), 4.6.2(b). Hence, by Proposition 3.4.1, $S(2 \mid 1, d)$ has wild type for $d \geq 2 p$. One can now apply Corollary 3.3.1(b) to conclude that $S(m \mid n, d)$ has wild representation type for $d \geq 2 p$.

\section{Global Dimension}

5.1 In [MZ, Conj. 1] it is conjectured that $S(m \mid n, d)$ is quasi-hereditary whenever $d$ is coprime to $p$. In this section we use our previous calculations to show this is far from true. In fact, we will prove the following theorem.

(5.1.1) Theorem . For $d \geq p \geq 5$ and $m, n \geq d$ the superalgebra $S(m \mid n, d)$ has infinite global dimension.

This result suggests the following question.

(5.1.2) Question . Is it true that $S(m \mid n, d)$ is either semisimple or has infinite global dimension? In particular, is it true that non-semisimple Schur superalgebras are never quasi-hereditary?

5.2 Before proving Theorem 5.1.1 we first need to determine which block of $k \Sigma_{d}$ contains a given signed Young module. Throughout the remainder of this section we assume $m, n \geq d$.

Recall the classification of irreducible $S(m \mid n, d)$-supermodules when $m, n \geq d$ given in [Do, $2.3(3)$ ] (for the statement when $m, n$, and $d$ are arbitrary, see [BK, Thm. 6.5]).

(5.2.1) Theorem . Let $m, n \geq d$. The irreducible supermodules of $S(m \mid n, d)$ are labeled by heighest weight by the set $\Lambda^{++}(m \mid n, d)=\{(\lambda \mid p \mu)|| \lambda|+p| \mu \mid=d\}$ where $\lambda$ and $\mu$ are partitions. 
Write $L(\lambda \mid p \mu)$ for the irreducible $S(m \mid n, d)$-supermodule of highest weight $(\lambda \mid p \mu)$ and let $P(\lambda \mid p \mu)$ denote the projective cover of $L(\lambda \mid p \mu)$.

Just as with the ordinary Schur algebra, one can define a Schur functor

$$
F: S(m \mid n, d) \text {-supermodules } \rightarrow k \Sigma_{d} \text {-modules. }
$$

Like with the ordinary Schur functor, $F$ is exact and if $L$ is an irreducible $S(m \mid n, d)$ -

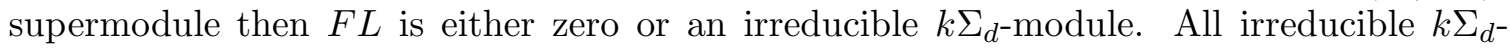
modules appear in this way. In fact, by [BK, (5.13)] we have

$$
F L(\lambda \mid p \mu) \cong \begin{cases}D^{m\left(\lambda^{\prime}\right)}, & \text { if }|\mu|=0 \text { and } \lambda^{\prime} \text { is } p \text {-regular } \\ 0, & \text { otherwise }\end{cases}
$$

Furthermore, by [Do, p. 662] we have

$$
F P(\lambda \mid p \mu) \cong Y^{(\lambda \mid p \mu)} .
$$

Thus to determine which block contains $Y^{(\lambda \mid p \mu)}$ it will suffice to obtain information about the composition factors of $P(\lambda \mid p \mu)$.

Before proceeding, we require additional notation. Let

$$
X=\left\{\xi=\left(\xi_{1}, \ldots, \xi_{m} \mid \xi_{m+1}, \ldots, \xi_{m+n}\right) \mid \xi_{1}, \ldots, \xi_{m+n} \in \mathbb{Z}\right\} .
$$

We view $(\lambda \mid p \mu) \in \Lambda^{++}(m \mid n, d)$ as an element of $X$ in the natural way as $(\lambda, 0, \ldots, 0 \mid p \mu, 0, \ldots, 0)$. For $i=1, \ldots, m+n$, let $\varepsilon_{i} \in X$ be the element which has a 1 in the $i$ th position and 0 's elsewhere. Let $\vartheta \in X$ be the element

$$
\vartheta=(-1,-2, \ldots,-m \mid m-1, m-2, \ldots, m-n+1, m-n) .
$$

Note that our definition of $\vartheta$ differs from the one in $[\mathrm{K},(2.8)]$ by a multiple of the $G L(m \mid n)$ analogue of the determinant representation. This has no significant effect on our arguments.

Given $i=1, \ldots, m+n$ we define $r_{i}: X \rightarrow \mathbb{Z}$ by

$$
r_{i}(\xi)= \begin{cases}\xi_{i}+\vartheta_{i}, & \text { if } i=1, \ldots, m ; \\ -\left(\xi_{i}+\vartheta_{i}\right), & \text { if } i=m+1, \ldots, m+n .\end{cases}
$$

For $r \in \mathbb{Z}_{p}$ and $\xi \in X$, define

$$
\begin{aligned}
& A_{r}(\xi)=\left|\left\{i=1, \ldots, m+n \mid r_{i}\left(\xi+\varepsilon_{i}\right) \equiv r(\bmod p)\right\}\right|, \\
& B_{r}(\xi)=\left|\left\{i=1, \ldots, m+n \mid r_{i}(\xi) \equiv r(\bmod p)\right\}\right| .
\end{aligned}
$$

For a partition $\lambda$ we let $\tilde{\lambda}$ denote its $p$-core. Recall that the blocks of $k \Sigma_{d}$ are parameterized by the $p$-cores of the partitions of $d$ [JK, Sect. 2.7]. Also recall that the category of $S(m \mid n, d)$-supermodules is equivalent to the category of polynomial representations of degree $d$ for the supergroup $G L(m \mid n)$. That is we can view the category of $S(m \mid n, d)$-supermodules as a full subcategory of the category of representations of $G L(m \mid n)$ and, in turn, as a full subcategory of the category of supermodules for the superalgebra $\operatorname{Dist}(G L(m \mid n))$ of distributions for $G L(m \mid n)$. See [BK] for a full discussion of these matters.

(5.2.4) Lemma . If $L(\lambda \mid p \mu)$ and $L(\nu \mid p \eta)$ have the same central character as $\operatorname{Dist}(G L(m \mid n))$ supermodules, then $\tilde{\lambda}=\tilde{\nu}$. 
Proof. If $L(\lambda \mid p \mu)$ and $L(\nu \mid p \eta)$ have the same central character, then by [K, Lem. 3.3] we have

$$
A_{r}(\lambda \mid p \mu)-B_{r}(\lambda \mid p \mu)=A_{r}(\nu \mid p \eta)-B_{r}(\nu \mid p \eta)
$$

for all $r \in \mathbb{Z}_{p}$. However, note that if $i=m+1, \ldots, m+n$, then

$$
\begin{aligned}
r_{i}(\lambda \mid p \mu) & \equiv-\vartheta_{i}(\bmod p) \\
& \equiv r_{i}(\nu \mid p \eta)(\bmod p),
\end{aligned}
$$

and

$$
\begin{aligned}
r_{i}\left((\lambda \mid p \mu)+\varepsilon_{i}\right) & \equiv-\vartheta_{i}-1(\bmod p) \\
& \equiv r_{i}\left((\nu \mid p \eta)+\varepsilon_{i}\right)(\bmod p) .
\end{aligned}
$$

Consequently, we see that (5.2.5) holds if and only if

$$
A_{r}^{\prime}(\lambda \mid p \mu)-B_{r}^{\prime}(\lambda \mid p \mu)=A_{r}^{\prime}(\nu \mid p \eta)-B_{r}^{\prime}(\nu \mid p \eta)
$$

for all $r \in \mathbb{Z}_{p}$, where for $\xi \in X$ we define

$$
\begin{aligned}
& A_{r}^{\prime}(\xi)=\left|\left\{i=1, \ldots, m \mid r_{i}\left(\xi+\varepsilon_{i}\right) \equiv r(\bmod p)\right\}\right|, \\
& B_{r}^{\prime}(\xi)=\left|\left\{i=1, \ldots, m \mid r_{i}(\xi) \equiv r(\bmod p)\right\}\right| .
\end{aligned}
$$

Given $(\lambda \mid p \mu)$ and $r \in \mathbb{Z}_{p}$, for brevity let us write $A_{r}^{\prime}$ for $A_{r}^{\prime}(\lambda \mid p \mu)$ and $B_{r}^{\prime}$ for $B_{r}^{\prime}(\lambda \mid p \mu)$. By definition, we have $A_{r}^{\prime}=B_{r-1}^{\prime}$ for all $r \in \mathbb{Z}_{p}$. We then have the following equations in the $B_{r}^{\prime}$ 's:

$$
\begin{aligned}
B_{1}^{\prime}+B_{2}^{\prime}+\cdots+B_{p}^{\prime} & =m \\
B_{r-1}^{\prime}-B_{r}^{\prime} & =: b_{r} \quad(\text { for } r=1, \ldots, p) .
\end{aligned}
$$

However, a straightforward check verifies that the determinant for this system of linear equations is nonzero so one can solve this system for the $B_{r}^{\prime}$ 's. That is, (5.2.6) holds if and only if

$$
B_{r}^{\prime}(\lambda \mid p \mu)=B_{r}^{\prime}(\nu \mid p \eta)
$$

for all $r \in \mathbb{Z}_{p}$. At this point we assume without loss that $|\lambda|=|\nu|$ since $B_{r}^{\prime}\left(\lambda, 1^{p} \mid p \mu\right)=$ $B_{r}^{\prime}(\lambda \mid p \mu)$ and since the $p$-cores of $\left(\lambda, 1^{p}\right)$ and $\lambda$ coincide.

Since for $i=1, \ldots, m$ we have $r_{i}(\lambda \mid p \mu)=\lambda_{i}-i$, it follows that (5.2.7) holds if and only if there is an element $\sigma \in \Sigma_{m}$ so that

$$
\lambda_{i}-i \equiv \nu_{\sigma(i)}-\sigma(i)(\bmod p)
$$

for all $i=1, \ldots, m$. Finally, by the Nakayama Rule [Mar, Thm. 5.1.1] we have that (5.2.8) holds if and only if $\tilde{\lambda}=\tilde{\nu}$. This proves the desired result.

(5.2.9) Corollary. The signed Young module $Y^{(\lambda \mid p \mu)}$ is in the $k \Sigma_{d}$ block with p-core $\tilde{\lambda}$.

Proof. Let $L(\nu \mid p \eta)$ be a composition factor of $P(\lambda \mid p \mu)$. Then since both $L(\lambda \mid p \mu)$ and $L(\nu \mid p \eta)$ are composition factors of an indecomposible $\operatorname{Dist}(G L(m \mid n))$-supermodule, they must have the same central characters (c.f. [K, Sect. 2.8]). By the previous lemma, we have that $\tilde{\lambda}=\tilde{\nu}$. The result then follows by exactness of the Schur functor along with (5.2.2) and $(5.2 .3)$. 
5.3 We can now prove Theorem 5.1.1. Since $p \geq 5$ there must be a partition $\tau \vdash d-p$ which is a $p$-core by the work of Granville and Ono [GO]. Consequently, $\Sigma_{d}$ has a $p$-block of defect one. Let $B$ denote this block.

(5.3.1) Lemma . There are $p+1$ signed Young modules in B. They are the $p$ distinct Young modules $Y^{(\lambda \mid \emptyset)} \cong Y^{\lambda}$ with $\tilde{\lambda}=\tau$ and one non-projective twisted Young module $Y^{(\tau \mid p)} \cong Y^{\left(\tau^{\prime}+p\right)} \otimes \operatorname{sgn}$.

Proof. By Corollary 5.2.9 the signed Young modules which occur in $B$ are those of the form $Y^{(\lambda \mid p \mu)}$ with $\tilde{\lambda}=\tau$. That is, precisely those listed. Now, since twisted Young modules are signed Young modules, we have $Y^{\left(\tau^{\prime}+p\right)} \otimes$ sgn is a signed Young module in the block $B$, and it must be $Y^{(\tau \mid p)}$.

Now we have the direct sum decomposition

$$
S(m \mid n, d)=\operatorname{End}_{k \Sigma_{d}}\left(V^{\otimes d}\right) \cong \operatorname{End}_{k \Sigma_{d}}\left(\bigoplus_{Y^{(\lambda \mid \mu)} \in B} a_{(\lambda \mid \mu)} Y^{(\lambda \mid \mu)}\right) \oplus \operatorname{End}_{k \Sigma_{d}}(U)
$$

where $a_{(\lambda \mid \mu)}$ denotes the multiplicity of $Y^{(\lambda \mid \mu)}$ in $V^{\otimes d}$ and $U$ has no summands in the block $B$. That is, we have direct sum decomposition of $S(m \mid n, d)$ into graded two-sided ideals, one of which is Morita equivalent to

$$
\operatorname{End}_{\Sigma_{d}}\left(\bigoplus_{Y^{(\lambda \mid \mu)} \in B} Y^{(\lambda \mid \mu)}\right)
$$

This is precisely the situation of (4.2.4) and the quiver is just $\tilde{\mathcal{A}}_{p}$. It is easy to see this (super)algebra has infinite global dimension. The projective resolutions of the simple modules are all periodic and do not terminate. Since a two-sided graded ideal has infinite global dimension, so does $S(m \mid n, d)$, proving Theorem 5.1.1.

\section{REFERENCES}

[A] S. Ariki, Hecke algebras of classical type and their representation type, preprint (2004).

[AM] S. Ariki, A. Mathas, The representation type of Hecke algebras of type B, Adv. Math. 181 (2004), 134-159.

[BN] B.D. Boe, D.K. Nakano, Representation type of Category $\mathcal{O}_{S}$, to appear in Adv. Math.

[BKI] J. Brundan, A. Kleshchev, Projective representations of symmetric groups via Sergeev duality, Math. Z. 239 (2002), no. 1, 27-68.

[BK] J. Brundan, J. Kujawa, A new proof of the Mullineux conjecture, J. Algebraic Combinatorics 18 (2003), 13-39.

[BKM] T. Brüstle, S. König, V. Mazorchuk, The coinvariant algebra and representation types of blocks of category $\mathcal{O}$, Bull. London Math. Soc. 33 (2001), no. 6, 669-681.

[CT] J. Chuang, K.M. Tan, On Young modules of defect 2 blocks of symmetric group algebras, J. Algebra 221 (1999), 651-668.

[CPS] E. Cline, B.J. Parshall, and L.L. Scott, Finite-dimensional algebras and highest weight categories, J. Reine Angew. Math. 391 (1988), 85-99.

[Do] S. Donkin, Symmetric and exterior powers, linear source modules and representations of Schur superalgebras, Proc. London Math. Soc. (3), 83 (2001), 647-680.

[DEMN] S.R. Doty, K. Erdmann, S. Martin and D.K. Nakano, Representation type of Schur Algebras, Math Z. 232 (1999),137-182. 
[DN] S.R. Doty, D.K. Nakano, Semisimple Schur algebras, Math. Proc. Camb. Phil. Soc. 124 (1998), no. 1, 15-20.

[DNP] S.R. Doty, D.K. Nakano and K.M. Peters, Infinitesimal Schur algebras of finite representation type, Quart. J. Math. Oxford (2), 48, (1997), 323-345.

[E] K. Erdmann, Schur algebras of finite type, Quart. J. Math. Oxford (2), 44, (1993), 17-41.

[EN1] K. Erdmann, D.K. Nakano, Representation type of $q$-Schur algebras, Trans. Amer. Math. Soc. 353 (2001), no. 12, 4729-4756.

[EN2] K. Erdmann, D.K. Nakano, Representation type of Hecke algebras of type A, Trans. Amer. Math. Soc. 354 (2002), 275-285.

[FNP] V. Futorny, D.K. Nakano, R.D. Pollack, Representation type of the blocks of category $\mathcal{O}$, Quart. J. Math. Oxford 52 (2001), no. 3, 285-305.

[GO] A. Granville, K. Ono, Defect zero $p$-blocks for finite simple groups, Trans. Amer. Math. Soc. 348 (1996), no. 1, 331-347

[HN] D.J. Hemmer, D.K. Nakano, Specht filtrations for Hecke algebras of type A, J. London Math. Soc. (2) 69 (2004), 623-638.

[J1] G.D. James, On the decomposition matrices of the symmetric groups. II, J. Algebra 43 (1976), 45-54.

[J2] G.D. James, The representation theory of the symmetric groups, Springer Lecture Notes 682, Berlin, Heidelberg, New York, 1978.

[JK] G.D. James, A. Kerber, The representation theory of the symmetric group, Addison-Wesley, London, 1981.

[K] J. Kujawa, Crystal structures arising from representations of $G L(m \mid n)$, submitted (2004).

[MZ] F. Marko, A.N. Zubkov, Schur superalgebras in characteristic $p$, to appear in Algebras and Representation Theory.

[Mar] S. Martin, Schur algebras and representation theory, Cambridge University Press, Cambridge, 1993.

[Mui] N. Muir, Polynomial representations of the general linear Lie superalgebras, PhD thesis, University of London, 1991.

[P] M.H. Peel, Hook representations of the symmetric groups, Glasgow Math. J. 12 (1971), 136-149.

[R] M.J. Richards, Some decomposition numbers for Hecke algebras of general linear groups, Math. Proc. Camb. Phil. Soc. 119 (1996), 383-402.

[S] J. Scopes, Symmetric group blocks of defect two, Quart. J. Math. Oxford (2) 46 (1995), 201-234.

Department of Mathematics, University of Toledo, 2801 W. Bancroft, Toledo, OH 43606 , USA

E-mail address: david.hemmer@utoledo.edu

Department of Mathematics, University of Georgia, Athens, GA 30602, USA

E-mail address: kujawa@math.uga.edu

Department of Mathematics, University of Georgia, Athens, GA 30602, USA

E-mail address: nakano@math.uga.edu 